\title{
Acute Lymphoblastic Leukemia in Childhood Following Pyoderma Gangrenosum
}

\author{
Aziza El Ouali1*, Manal Azizi', Nadia Bouhafs', Mounia Rimani², Ayad Ghannam¹, \\ Abdeladim Babakhouya', Maria Rkain'1, Noufissa Benajiba1 \\ ${ }^{1}$ Hematology and Oncology Unit, Departments of Pediatric, UHC Mohammed VI, Medical School of OUJDA, University \\ Mohamed I, Oujda, Morocco \\ ${ }^{2}$ Hassan Pathological Anatomy Center, Rabat, Morocco \\ Email: *azizaelouali2@gmail.com
}

How to cite this paper: El Ouali, A., Azizi, M., Bouhafs, N., Rimani, M., Ghannam, A., Babakhouya, A., Rkain, M. and Benajiba, N. (2020) Acute Lymphoblastic Leukemia in Childhood Following Pyoderma Gangrenosum. Journal of Cancer Therapy, 11, 715-721.

https://doi.org/10.4236/jct.2020.1111060

Received: May 27, 2020

Accepted: November 20, 2020

Published: November 23, 2020

Copyright $\odot 2020$ by author(s) and Scientific Research Publishing Inc. This work is licensed under the Creative Commons Attribution International License (CC BY 4.0).

http://creativecommons.org/licenses/by/4.0/

\begin{abstract}
Pyoderma gangrenosum (PG) is a rare but serious ulcerating skin disease that occurs either idiopathically or associated with various systemic diseases and malignant tumors. Although the association of PG with myeloid malignant tumors is well known, their association with lymphoid malignant tumors, especially in children is extremely rare. We present here a case of PG in a 4-year and 4 months female child, diagnosed in the Pediatrics department, which had initially evolved well with oral corticosteroid therapy and a local dermocorticoid. The development was marked 7 months after stopping corticosteroid treatment by the occurrence of acute lymphoblastic leukemia. This case of PG is presented in the intention of increasing awareness of this uncommon condition with may occurring in association with haematological malignancy. PG may precede, develop concurrently with, or follow various conditions.
\end{abstract}

\section{Keywords}

Pyoderma Gangrenosum, Childhood, Leukemia

\section{Introduction}

Pyoderma gangrenosum is a rare, ulcerative, cutaneous condition, which was first described in 1930, by Brun-Sting et al. [1]. It usually occurs between the ages of 25 and 54 and rarely affects children. It is most commonly seen in patients with chronic inflammatory bowel disease, gammopathies [2] [3], rheumatoid arthritis, diabetes mellitus, myelodysplastic syndrome and myeloid leukemia. Although the association of PG myeloid malignancies is well known, its association with lymphoid malignancies, particularly in children is ex- 
tremely rare. We report late discovery of acute lymphoblastic leukemia in the case of a 4-year and 4 months female child exmined in Pediatrics department for a PG who had evolved well under Prednisone at a maintenance dose of $5 \mathrm{mg} / \mathrm{d}$. The evolution was marked by the occurrence of fever, pallor and bleeding manifestations, a consistent with diagnosis of acute lymphoblastic leukemia.

\section{Case Report}

A four year 4 months old female child presented with fever $\left(39^{\circ} \mathrm{C}\right)$, progressive pallor, lethargy, of five days duration associated with ulcerated skin lesion in the right arm .The lesion had initially started as a small erythematous lesion which progressed to a necrotic. On physical examination, she had marked pallor with fever. She had no bleeding manifestations or lymphadenopathy or hepatosplenomegaly. Cutaneous examination revealed a deep ulceration of $4 \mathrm{~cm}$ on the right arm with a violaceous border that overhangs the ulcer bed and virulent exudate at the ulcer base. There were also one other ulceration of $2 \mathrm{~cm}$ on the left leg very painful (Figure 1, Figure 2). She had normochromic normocytic anemia at $5.5 \mathrm{~g} / \mathrm{dl}$, white blood cell count of $1680 / \mathrm{mm}^{3}$, neutropenia at $710 \mathrm{~mm}^{3}$,

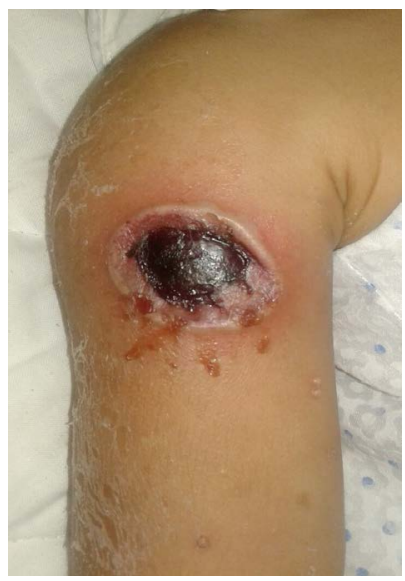

Figure 1. Deep ulceration of $4 \mathrm{~cm}$ on the right arm with a violaceous border that overhangs the ulcer bed, and purulent exudate at the ulcer base.

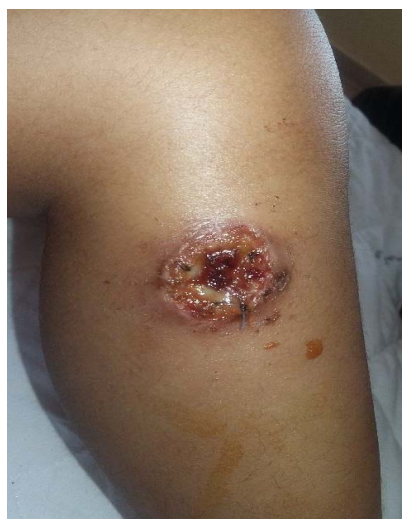

Figure 2. $2 \mathrm{~cm}$ ulcer of the left leg presenting violaceous borders overhanging the ulcer bed with serosanguinous exudates at the ulcer base with fibrinous tissue. 
lymphopenia at $650 / \mathrm{mm}^{3}$ and platelets count of $23,000 / \mathrm{mm}^{3}$. She was started on broad-spectrum antimicrobials in view of febrile neutropenia (third generation of cephalosporins + amikacine). Bone marrow aspiration done in view of pancytopenia was suggestive of poor cellularity. No abnormalities were observed on the peripheral blood smear. The bone marrow biopsy was normal. The C-reactive protein was high at $370 \mathrm{mg} / \mathrm{l}$, procalcitonin at $13.21 \mathrm{ng} / \mathrm{ml}$, erythrocyte sedimentation right at $145 \mathrm{~mm}$ and ferritinemia at $612 \mathrm{~g} / \mathrm{l}$. Viral serology including HIV, hepatitis B and C, EBV, and CMV were negative. Evaluation of her immune system showed a normal igA, IgG, IgM, and IgE levels. Diagnosis of Hemophagocytic syndrome has been ruled out. Cultures for bacteria and fungi from skin lesions, and blood cultures were repeatedly negative. Dermatology consultation was obtained for the arm and legs ulcer, and a probability of PG was considered. The histological study revealed pyoderma with spongiosis and vesiculation mainly harboring neutrophils with vascular thrombosis and extensive hypodermic ischemic necrosis (Figure 3, Figure 4), without malignant tumor

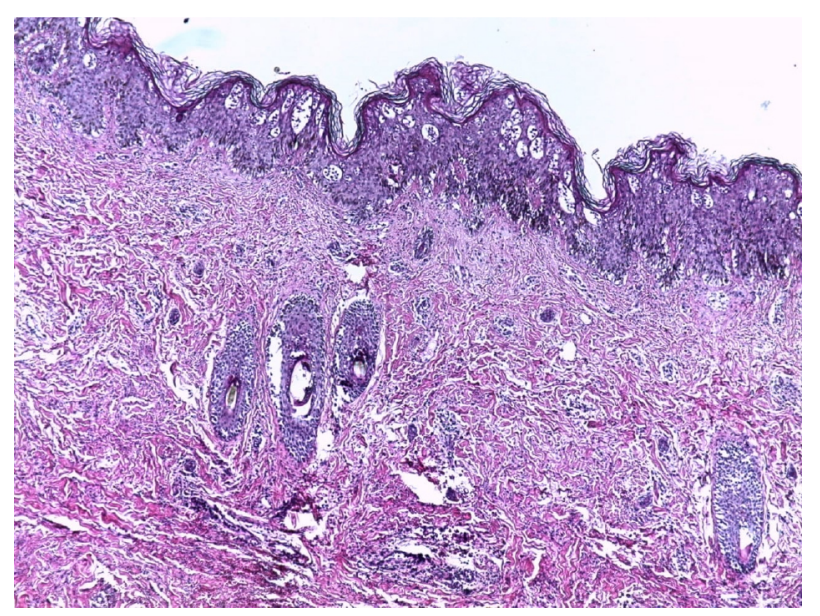

Figure 3. Microphotography showing extensive spongiosis of the epidermis with infiltration of the superficial dermis by chronic and acute inflammatory cells. (HE, 100 $\times$ ).

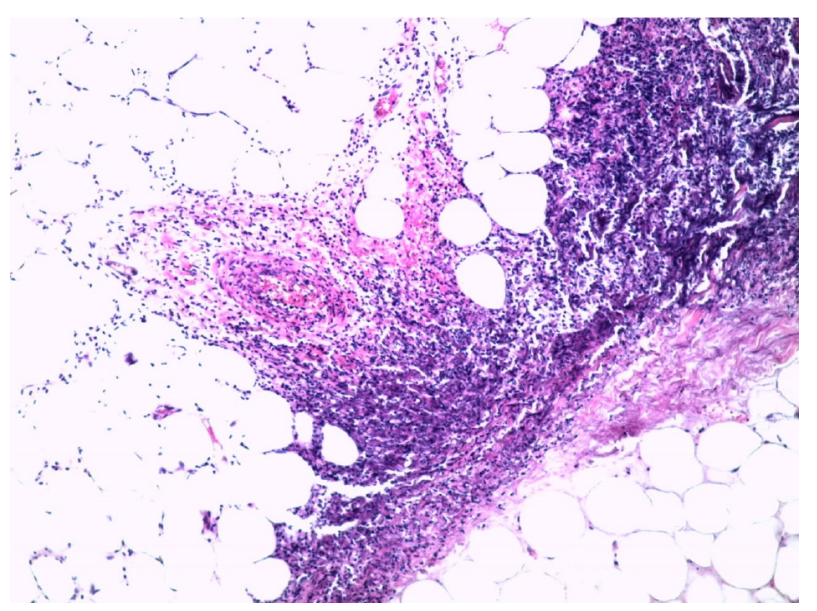

Figure 4. Microphotography showing inflammation extending to the aubcutis with leucocytoclastic vasculitis (HE, 200×). 
process, nor of tuberculoid granuloma. She was started on prednisone and a local dermocorticoid. Within 5 days of starting treatment patient improved symptomatically. Ulcer started healing with improvement of the hemogram; hemoglobin increased to $8 \mathrm{~g} / \mathrm{dl}$, white blood cells at $3250 / \mathrm{mm}^{3}$, lymphocytes at $2230 / \mathrm{mm}^{3}$, polynuclear neutrophils (PNN) still low at PNN at $740 / \mathrm{mm}^{3}$. The child was followed regularly in a hematology and oncology unit. Within 25 days of starting treatment corticosteroids ulcer healed completely (Figure 5, Figure 6). After 7 months of stopping corticosteroid therapy, the child was readmitted to pallor, fever and bleeding manifestations. She had hemoglobin of $4.8 \mathrm{~g} / \mathrm{dl}$, white blood cell of $20,320 / \mathrm{mm}^{3}$, and platelet of $18,000 / \mathrm{mm}^{3}$, blood smear suggest of $87 \%$ the blasts. Bone marrow aspiration done in view of pancytopenia was suggestive of ALL and flow-cytometry revealed expression of CD10, CD19, CD22, thus confirming the diagnosis of acute lymphoblastic leukemia associated-positive B-cell ALL. Chemotherapy according to the Marall 2006 protocol was started with good progress. The child is currently in the phase of maintenance therapy.

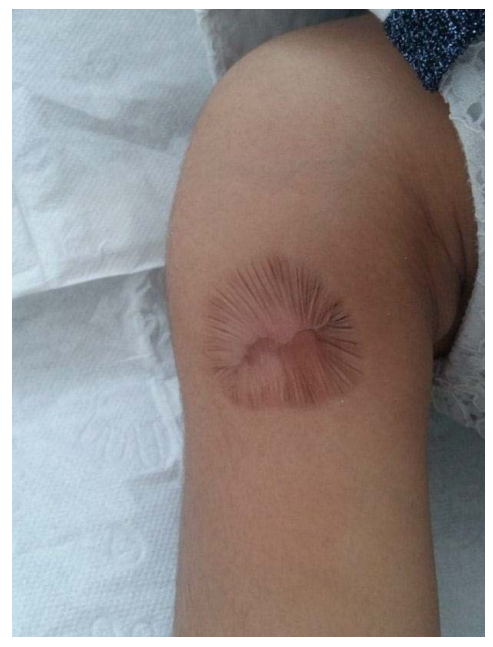

Figure 5. Evolution of the ulceration.

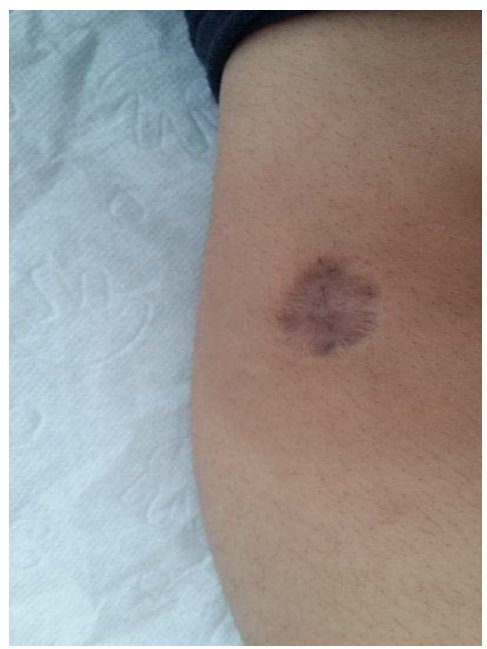

Figure 6. Evolution of the ulceration. 


\section{Discussion}

Pyoderma gangrenosum is a rare but serious ulcerative skin disorder afflicting both adults and children. Her precise incidence is difficult to ascertain. At the Mayo clinic, 180 cases were diagnosed in 53 years and $4 \%$ of these were aged under 15 years [2] [3]. The aetiology and pathogenesis is poorly understood, but it is thought to arise because of an underlying immune dysregulation. The diagnosis is often delayed and only considered after eliminating other processes that may cause cutaneous ulcers and is only supported by histopathology. Criteria for diagnostic had been proposed for PG [4]. Our patient fulfills these criteria. The differential diagnosis of ulcerative cutaneous lesions include infectious disease, malignancy, vasculitis, insect bites, venous or arterial insufficiency (including antiphospholipid antibody associated occlusive disease), and factitious (self-inflicted) ulcerations [5]. There are five clinical variants of PG: ulcerative/classical, bullous, pustular, peristomal, and vegetative [6]. Bullous pyoderma gangrenosum is a superficial variant that affects the upper limbs and face more than the lower limbs. It has been reported with haematological disease, such as preleukaemic conditions (i.e. myeloid metaplasia) and acute myelogenous leukaemia [7] [8]. The distribution of lesions is similar to that in adults, often involving the lower extremities, but PG of the head and face appears to be more common in children [9] [10]. Most patients with PG have multiple lesions, as in our patient, although the exact etiology and pathogenesis are poorly understood. Approximately $50 \%$ of patients with PG have an associated systemic disease. These diseases may precede, occur simultaneously, or follow [10] [11], as in our patient. In children, it usually occurs in association with inflammatory bowel disease, immunodeficiency, immunosuppression, and HIV infection [8]. Maldonado et al. first reported the occurrence of PG with leukemia [12]. Leukemia is the most frequently associated malignancy, with acute myeloid leukemia being the most common form [13]. PG in lymphoid leukemia is rare. Our patient had this rare association. In such cases, the child commonly presents with general status alteration with hepatosplenomegaly and palpable lymph nodes on physical examination, a complete blood count, lood smear, and bone marrow aspiration are to be done. No specific therapy is universally effective for patients with PG. Effective control of the underlying disease may be linked to a control of the cutaneous process as well [14]. The most frequently prescribed treatment for children is, topical therapy and systemic corticosteroids, which generally are very effective [6]. Our child responded to oral prednisolone. Other agents used are immunosuppressive agents such as cyclosporine, tumor necrosis factor-alpha blockers such as infliximab, adalimumab, and biologics such as efalizumab and alefacept [6] [14]. In patients without an identifiable associated disease, it is still possible for it to appear later; hence follow-up and evaluation are required even after the skin lesions have healed [15].

\section{Conclusion}

Pyoderma gangrenosum is often misdiagnosed and mismanaged. Therefore cli- 
nicians must have a large index of suspicion, particularly in children with a rapidly progressive or non healing cutaneous ulcer. An exhaustive search should be made for any associated disorder and permanent surveillance will be required. The association with leukemia may be more common than previously thought, and that surveillance of leukemias should be maintained in Atypical cases of PG to enable early diagnosis and appropriate treatment. The present work shows that pyoderma gangrenosum can be an early manifestation of ALL or LBL in childhood.

\section{Conflicts of Interest}

The authors declare no conflicts of interest regarding the publication of this paper.

\section{References}

[1] Brunsting, L.A., Goeckerman, W.H. and O'Leary, P.A. (1930) Pyoderma (Echthyma) Gangrenosum: Clinical and Experimental Observations in Five Cases Occurring in Adults. Archives of Dermatologie, 22, 655-680. https://doi.org/10.1001/archderm.1930.01440160053009

[2] Powell, F.C. and Perry, H.O. (1984) Pyoderma Gangrenosum in Childhood. Archives of Dermatology, 120, 757-761. https://doi.org/10.1001/archderm.1984.01650420067018

[3] Powell, F.C., Schroeter, A.L., Su, W.P. and Perry, H.O. (1985) Pyoderma Gangrenosum: A Review of 86 Patients. Quarterly Journal of Medicine, 55, 173-186.

[4] Su, W.P.D., Davis, M.D.P., Weenig, R.H., Powell, F.C. and Perry, H.O. (2004) Pyoderma Gangrenosum: Clinicopathologic Correlation and Proposed Diagnostic Criteria. International Journal of Dermatology, 43, 790-800.

https://doi.org/10.1111/j.1365-4632.2004.02128.x

[5] Callen, J.P. (1998) Pyoderma Gangrenosum. Lancet, 351, 581-585. https://doi.org/10.1016/S0140-6736(97)10187-8

[6] Miller, J., Yentzer, B.A., Clark, A., Jorizzo, J.L. and Feldman, S.R. (2010) Pyoderma Gangrenosum: A Review and Update on New Therapies. Journal of the American Academy of Dermatology, 62, 646-654. https://doi.org/10.1016/j.jaad.2009.05.030

[7] Brooklyn, T., Dunnill, G. and Probert, C. (2006) Diagnosis and Treatment of Pyoderma Gangrenosum. BMJ, 333. https://doi.org/10.1136/bmj.333.7560.0-c

[8] Kamile, A., Yasemin, Ö. and Mehmet, C. (2017) Bullous Pyoderma Gangrenosum as the Presenting Sign of Acute Myeloid Leukemia in a Child. Journal of Pediatric Hematology/ Oncology, 39, 312-313. https://doi.org/10.1097/MPH.0000000000000796

[9] Graham, J.A., Hansen, K., Rabinowitz, L.G. and Esterly, N.B. (1994) Pyoderma Gangrenosum in Infants and Children. Pediatric Dermatology, 11, 10-17. https://doi.org/10.1111/j.1525-1470.1994.tb00065.x

[10] Bakhshi, S., Sethuraman, G., Singh, M.K. and Singh, L.A. (2005) Atypical Pyoderma Gangrenosum as a Manifestation of Childhood Acute Lymphoblastic Leukemia. Pediatric Dermatology, 22, 543-545. https://doi.org/10.1111/j.1525-1470.2005.00136.x

[11] Prystowsky, J.H., Kahn, S.N. and Lazarus, G.S. (1989) Present Status of Pyoderma Gangrenosum: Review of 21 Cases. Archives of Dermatology, 125, 57-64. https://doi.org/10.1001/archderm.1989.01670130059007 
[12] Powell, F.C., Su, W.P. and Perry, H.O. (1996) Pyoderma Gangrenosum: Classification and Management. Journal of the American Academy of Dermatology, 34, 395-409. https://doi.org/10.1016/S0190-9622(96)90428-4

[13] Maldonado, N., Torres, V.M., Méndez-Cashion, D., Pérez-Santiago, E. and Cáceres de Costas, M. (1968) Pyoderma Gangrenosum Treated with 6-Mercaptopurine and Followed by Acute Leukemia. The Journal of Pediatrics, 72, 409-414. https://doi.org/10.1016/S0022-3476(68)80218-5

[14] Loganathan, A., Srinivasan, A., Scott, J.X. and Ramamoorthy, R. (2019) Pyoderma Gangrenosum in Childhood Acute Lymphoblastic Leukemia. Indian Journal of Paediatric Dermatology, 20, 157-159.

https://doi.org/10.4103/ijpd.IJPD 5918

[15] Chow, R.K.P. and Ho, V.C. (1996) Treatment of Pyoderma Gangrenosum. Journal of the American Academy of Dermatology, 34, 1047-1060.

https://doi.org/10.1016/S0190-9622(96)90285-6 